\title{
CELL SIZE IN HYBRID WIRELESS SYSTEMS
}

\author{
Tamer A. ElBatt, Anthony Ephremides \\ Electrical Engineering Department, \\ University of Maryland, \\ College Park, MD 20742, USA. \\ e-mail: telbatt@eng.umd.edu, tony@eng.umd.edu
}

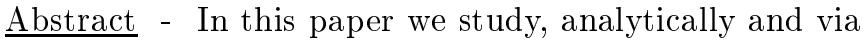
simulation, the impact of reducing the cell size of a wireless system on the system capacity and hand-off failure rate. First, we focus on a pure cellular system. We develop exact and approximate models for cellular systems in order to emphasize the blocking probability/forced termination probability trade-ff. This trade-off motivates the problem of optimizing the system performance with respect to the cell size. For hybrid systems, we investigate how jointly optimizing the number of cells per spot-beam, and the bandwidth partitioning could improve the QoS parameters of interest. For both systems, the numerical solution is only feasible for small number of cells. For large systems, a simulation study is presented.
\end{abstract}

\section{INTRODUCTION}

The expected increase in demand for mobile communication services calls for efficient use of the communication bandwidth. Various approaches have been introduced in the literature for increasing the capacity of cellular systems. Reducing the frequency reuse factor, via cell sectorization, and reducing the cell size[1],[2] are among the well known techniques. Both techniques permit denser frequency reuse patterns. This is achieved at the expense of higher rate of cell-boundary crossings, higher complexity of antenna design, and the need for additional frequency planning.

In [3], the authors applied the concept of cluster planning, via which the proposed sectoring arrangement allows microcells to reuse macrocell frequencies. This was shown to achieve higher system capacity. However, users' mobility and hand-offs were not considered in that model.

This work is along the same line of the work done in [4]. We concluded that a satellite/cellular hybrid system is superior to the pure satellite and pure cellular extremes. Here, we study the same system from a different perspective. We investigate the impact of reducing the cell size on the system capacity and hand-off failure rate. As earlier studies indicated, smaller cells permit denser frequency reuse patterns and hence improve the system capacity. Later, we show that the price paid is two fold, the increase in the forced termination probability and the signaling and processing overhead necessary for handling frequent hand-off attempts.
The paper is thus organized as follows: In section II, exact and approximate models for a pure cellular system are developed. The satellite/cellular hybrid system is studied in section III. Finally, the conclusions are drawn in section IV.

\section{PURE CELLULAR SYSTEMS}

In order to study a pure cellular system, we first make the following assumptions and introduce appropriate notation. New calls arrive at cell $C_{i}$ according to a Poisson arrival process with rate $\lambda$ calls/min. The duration of each call is assumed to be exponentially distributed with mean $1 / \mu$ min. The network under consideration is assumed to consist of $\mathrm{n}$ hexagonal cells, namely $C_{1}, C_{2}, \ldots$, and $C_{n}$. We define $K_{c}$ as the cellular reuse factor, that is the number of cells per cluster. The total number of pre-design resources available to the system is $\mathrm{M}$, where,

$$
\mathrm{M}=\Sigma_{i=1}^{K_{c}} \mathrm{M}_{i}
$$

and $M_{i}$ is the number of channels dedicated to $C_{i}$.

The base stations, namely $B S_{i}, \mathrm{i}=1, \ldots, \mathrm{n}$, communicate via a terrestrial wireline infrastructure. All call types have the same priority and all calls considered in this model are mobile-to-fixed calls. The inter-handoff time for a mobile is assumed to be exponentially distributed with mean $1 / \lambda_{h}$ min. This assumption is valid only for macrocells where the hand-off events are assumed to be independent. Finally, we assume that blocked calls are cleared.

The chosen QoS parameters should reflect accurately the impact of reducing the cell size on both the system capacity and the hand-off failure rate. For hand-off failures, we have two candidate QoS parameters, namely the dropping probability and the forced termination probability. The dropping probability is defined as the fraction of hand-off attempts that are denied access due to lack of resources. On the other hand, the forced termination probability is defined as the probability that a call accepted to the system is interrupted, during its lifetime, due to hand-off failure. In this paper, we consider both criteria and show the difference between their behavior when the cell size is reduced.

\section{A. Exact Model}


A pure cellular system consisting of 2 hexagonal cells can be modeled as a 2-dimensional continuous-time Markov Chain. It can be easily verified that there are diagonal state transitions caused by mobile users' handoff. Consequently, this Markov Chain is not a pure birthdeath process, and thus the product-form solution turns out to be inapplicable. For this model, the only feasible way to determine the QoS parameters is to solve the global balance equations. In a similar fashion, an ndimensional Markov Chain based model was developed for cellular systems having $\mathrm{n}=3,4,5$ hexagonal cells.

The effect of reducing the cell size (or equivalently increasing the number of cells in the specified geographical area) has been determined by solving the global balanace equations numerically for the set of parameters given in Table 1. In addition, the following two assumptions need to be made:

- The total call arrival rate to the covered area is assumed to be fixed. Thus, the call arrival rate per cell is inversely proportional to the number of cells in the covered area.

- The hand-off rate is assumed to be inversely proportional to the cell radius. This implies that it is directly proportional to the square root of the number of cells in the covered area [6].

Table 1. System Parameters

\begin{tabular}{|l|l|}
\hline Total System Bandwidth $(\mathrm{M})$ & 2 channels \\
\hline \# Cells in the covered area $(\mathrm{n})$ & $2,3, . ., 5$ cells \\
\hline Call Arrival Rate per Cell $(\lambda)$ & $\frac{1.6}{n}$ calls $/ \mathrm{min}$ \\
\hline Call Service Rate $(\mu)$ & 0.8 calls $/ \mathrm{min}$ \\
\hline Cellular Reuse Factor $\left(K_{c}\right)$ & 2 \\
\hline Call Hand-off Rate for $\mathrm{n}=2\left(\lambda_{h}\right)$ & 0.08 calls $/ \mathrm{min}$ \\
\hline
\end{tabular}

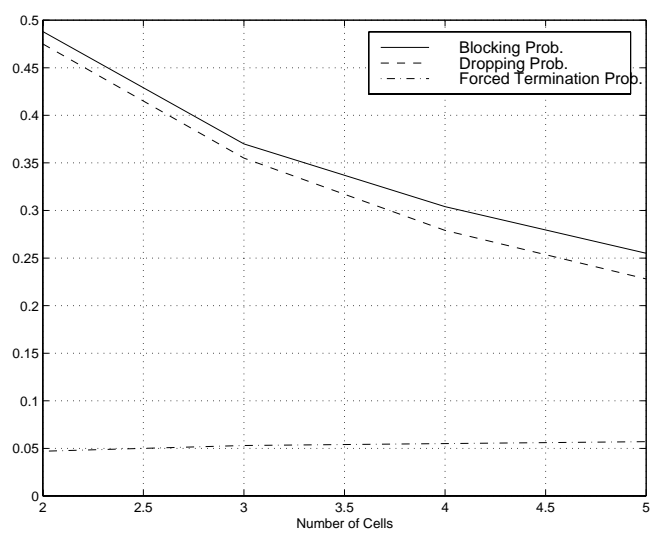

Figure 1. Qos Parameters vs. Number of Cells in the covered area : Numerical Results

It can be noticed from Figure 1 that the blocking and dropping probabilities are of the same order and have the same behavior. They both decrease for $\mathrm{n}>K_{c}$. Second, the forced termination probability is one order of magnitude lower than the dropping probability, and this is due to the low mobility pattern assumed. Third, the forced termination probability increases as the number of cells in the given area increases. These results emphasize the trade-off between the blocking probability and the forced termination probability as the number of cells increases.

\section{B. Approximate Model}

In order to develop a formal analysis for the behavior of the QoS parameters as the number of cells increases, we need to make the following approximations. Consider the simple 2-cell system modeled earlier. First, each hand-off event is approximated by arrival and departure events from respective cells. Under this approximation, the 2dimensional Markov chain becomes pure birth-death process with state-dependent arrival rates as given in the following equations,

$$
\begin{aligned}
& \lambda_{1}\left(x_{2}\right)=\left\{\begin{array}{rll}
\lambda+\left(x_{2}+1\right) \lambda_{h} & : & 0 \leq x_{2} \leq M_{2}-1 \\
\lambda & : & x_{2}=M_{2}
\end{array}\right. \\
& \lambda_{2}\left(x_{1}\right)=\left\{\begin{array}{rll}
\lambda+\left(x_{1}+1\right) \lambda_{h} & : & 0 \leq x_{1} \leq M_{1}-1 \\
\lambda & : & x_{1}=M_{1}
\end{array}\right.
\end{aligned}
$$

where,

$\lambda_{1}=$ Aggregate call arrival rate (new + hand-off) to $C_{1}$. $\lambda_{2}=$ Aggregate call arrival rate (new + hand-off) to $C_{2}$. $x_{i}=$ Number of active calls in cell $C_{i}, \mathrm{i}=1,2$.

In addition, the aggregate call departure rate is given by,

$$
\mu_{t}=\mu+\lambda_{h}
$$

Nevertheless, the detailed balance equations do not hold and thus the product-form solution remains inapplicable even under this approximation. To circumvent the state-dependent arrival rates hurdle, we approximate the number of active calls by their expected values. This is done to facilitate the mathematical analysis. Later, we will justify this approximation via comparing the approximate results to the exact ones. Accordingly, we get the following expressions for $\lambda_{1}$ and $\lambda_{2}$,

$$
\begin{aligned}
& \lambda_{1}=\lambda+E\left[x_{2}\right] \lambda_{h} \\
& \lambda_{2}=\lambda+E\left[x_{1}\right] \lambda_{h}
\end{aligned}
$$

Based on the aforementioned approximations, the detailed balance equations hold and the product-form solution becomes applicable. Accordingly, the system steadystate probabilities can be written in the following form,

$$
P\left(x_{1}, x_{2}\right)=\frac{1}{G} \cdot \frac{\rho_{1}^{x_{1}}}{x_{1} !} \cdot \frac{\rho_{2}^{x_{2}}}{x_{2} !} \quad, 0 \leq x_{i} \leq M_{i}, i=1,2
$$

where ' $G$ ' is the normalization constant and,

$$
\rho_{1}=\frac{\lambda_{1}}{\mu_{t}} \quad, \quad \rho_{2}=\frac{\lambda_{2}}{\mu_{t}}
$$

The next step is to compute the system blocking and dropping probabilities using this model. According to the assumptions, the spatial symmetry of the call arrival rates, service rates, and hand-off rates among the cells can be noticed. Moreover, if $M_{1}=M_{2}=\frac{M}{2}$, then it can be shown that $\rho_{1}=\rho_{2}=\rho$. Accordingly, it can be shown that the system blocking probability is given by,

$$
P_{b}=\operatorname{Er}\left(\rho, \frac{M}{2}\right)
$$


where $\operatorname{Er}\left(\rho, \frac{M}{2}\right)$ is the Erlang-B function [7].

Likewise, the dropping probability is found to be given by the same expression in (1). The forced termination probability $\left(P_{f}\right)$ can be derived from $P_{d}$ as in [5]. This model was used to compute the QoS parameters using the parameters given in Table 1. Figure 2 shows good agreement between the results of the exact model and the approximate one.

The previous analysis can be directly extended to larger systems having $\mathrm{n}>2$ cells. It can be shown that the blocking probability is given by the following expression,

$$
P_{b}=\operatorname{Er}\left(\rho, \frac{M}{K_{c}}\right)
$$

where,

$$
\rho=\frac{\lambda+E[x] \lambda_{h}}{\left(\mu+\lambda_{h}\right)}
$$

and $\mathrm{E}[\mathrm{x}]$ is the expected number of active calls in any cell.

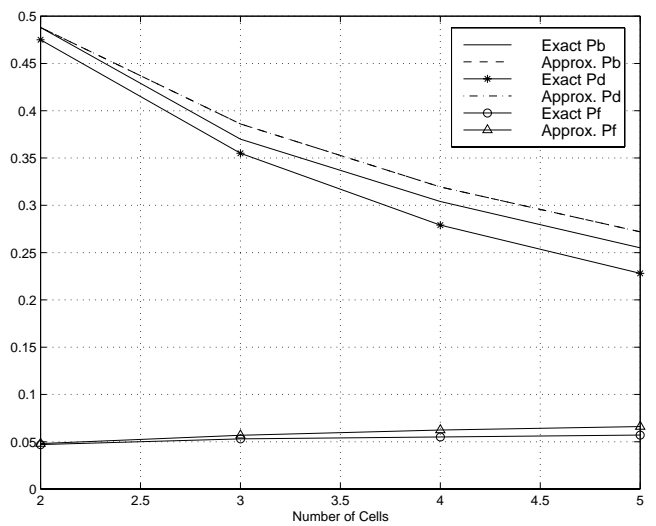

Figure 2. Exact and Approximate behavior of $P_{b}$, $P_{d}$ and $P_{f}$ versus $\mathbf{n}$

\section{Cell Size Impact on the QoS Parame- ters}

Our objective in this section is to show, analytically, the behavior of the QoS parameters as the cell size is reduced. First, we show how the blocking probability varies with the number of cells 'n'. The rate of variation of the blocking probability with $\mathrm{n}$ can be determined from the following equation,

$$
\frac{\partial P_{b}}{\partial n}=\frac{\partial P_{b}}{\partial \rho} \frac{\partial \rho}{\partial n}
$$

To determine the behavior of $P_{b}$ as $\mathrm{n}$ increases, it is necessary to check the trends of $P_{b}$ and $\rho$ via examining the signs of their partial derivatives in the right hand side of (3). Details of the derivation are ommited due to space limitations. It can be shown that,

$$
\frac{\partial P_{b}}{\partial n}<0 \quad, n \geq K_{c}
$$

It can be noticed from (4) that, for $n \geq K_{c}$, the blocking probability decreases as 'n' increases. Recall that, under the previous approximation, the dropping probability is also given by the Erlang-B function. Therefore, the dropping probability also decreases as 'n' increases. Finally, it is worth mentioning that these trends agree with those obtained earlier using the exact model.

The next step is to investigate the behavior of the forced termination probability as ' $n$ ' increases. For exponentially distributed call durations, and exponentially distributed inter-handoff times, the forced termination probability is shown to be[5],

$$
P_{f}=\frac{\lambda_{h} P_{d}}{\mu+\lambda_{h} P_{d}}
$$

The following definition is made to simplify the expressions,

$$
y=\lambda_{h} P_{d}
$$

Accordingly, the rate of variation of $P_{f}$ with respect to $\mathrm{n}$ can be written as,

$$
\frac{\partial P_{f}}{\partial n}=\frac{\partial P_{f}}{\partial y} \frac{\partial y}{\partial n}
$$

and the problem now amounts to checking the behavior of $\mathrm{y}$ as $\mathrm{n}$ increases. It can be shown that,

$$
\begin{aligned}
\frac{\partial y}{\partial n}=\frac{\lambda_{H}}{\sqrt{n}} & \operatorname{Er}(\rho, N)[0.5 \\
& \left.+n \frac{\partial \rho}{\partial n} \frac{N}{\rho}\left(1-\frac{\operatorname{Er}(\rho, N)}{\operatorname{Er}(\rho, N-1)}\right)\right]
\end{aligned}
$$

where $\lambda_{H}$ is a constant and $\mathrm{N}=\frac{M}{K_{c}}$.

From (7), it can be noticed that the sign of $\frac{\partial y}{\partial n}$ depends on $\frac{\partial \rho}{\partial n}$, which is negative, along with the load $\rho$, the number of cells n, and the number of resources N. Assume sufficiently large number of resources per cell $(\mathrm{N} \geq 10)$. As shown in Figure 3, we can divide the load range to the following three intervals,

(i) $\rho<0.4 \mathrm{~N}$, which is considered light load.

(ii) $0.4 \mathrm{~N} \leq \rho \leq 7 \mathrm{~N}$, which is considered intermediate load.

(iii) $\rho>7 \mathrm{~N}$, which is considered heavy load.

For light and heavy loads, it can be noticed from Figure 3 , that $\frac{\partial \operatorname{Er}(\rho, N)}{\partial \rho} \leq 0.002$, and hence $\operatorname{Er}(\rho, \mathrm{N}) \approx \operatorname{Er}(\rho$, $\mathrm{N}-1$ ). For these intervals, it follows immediately from (7) that $\frac{\partial y}{\partial n}>0$. Thus it can be concluded that under light and heavy load conditions the forced termination probability increases as 'n' increases. For intermediate loads, it is not clear that the same assertion can be made. It is worth mentioning here that real systems are designed to operate in the load range that achieves $1 \%$ blocking probability, which corresponds to light load conditions. 


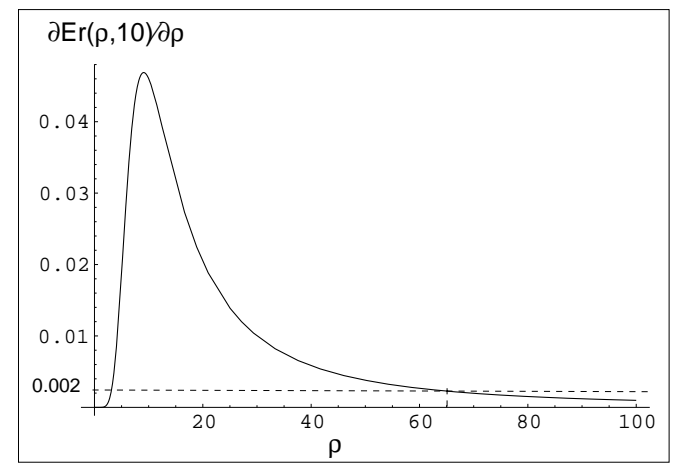

Figure 3. $\frac{\partial E r(\rho, N)}{\partial \rho}$ for $\mathbf{N}=\mathbf{1 0}$

\section{The Optimization Problem}

In this simulation study, we employ a more realistic mobility model. This model assumes the speed of mobile users to be uniformly distributed between maximum and minimum values. Moreover, the direction of movement is assumed to be uniformly distributed between 0 and $2 \pi$. Various network setups were simulated and tested using the numerical parameters shown in Table 2 .

Table 2. Numerical Parameters for the Simulation Model

\begin{tabular}{|l|l|}
\hline Total System Bandwidth $(\mathrm{M})$ & 100 channels \\
\hline \# Cells in the covered area $(\mathrm{n})$ & $50, \ldots, 400$ cells \\
\hline Total Call Arrival Rate & 0.33 calls $/ \mathrm{min}$ \\
\hline Call Service Rate $(\mu)$ & 0.33 calls $/ \mathrm{min}$ \\
\hline Cellular Reuse Factor $\left(K_{c}\right)$ & 7 \\
\hline Minimum Speed & $12 \mathrm{mph}$ \\
\hline Maximum Speed & $58 \mathrm{mph}$ \\
\hline
\end{tabular}

It can be noticed from Figure 4 that the trends of the QoS parameters agree with those obtained earlier using the exact and approximate models.

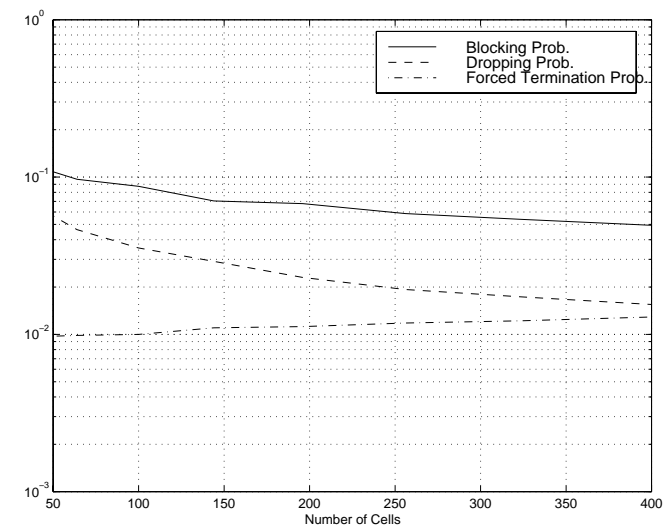

Figure 4. QoS Parameters vs. Number of Cells for a Pure Cellular System: Simulation Results

The optimum number of cells in the given area is ob- tained via solving the following minimization problem:

$$
\min _{n}\left(P_{b}+\alpha . P_{f}\right)
$$

where $\alpha$ is a weighting factor.

The following formulation is equivalent and easier to implement,

$$
\min _{n} P_{b}
$$

s.t.

$$
P_{f} \leq \beta
$$

The quantity $\beta$ is the alternative (equivalent) parameter in a one-to-one correspondence to the value of $\alpha$.

It is clear that as ' $n$ ' increases, the dimensionality of the Markov chain increases. Therefore, we had to resort to simulation. Assume that the parameter $\beta$ in (9) is set to 0.01 . From Figure 4, it can be noticed that, under this constraint, the minimum achievable blocking probability is 0.087 . This is achievable via deploying 100 hexagonal cells in the area of interest.

\section{SATELLITE/CELLULAR HYBRID SYSTEMS}

In order to study a hybrid system, assumptions similar to those of the pure cellular system are made. In addition, we assume that there is one satellite emitting 4 spot-beams covering the same area. Define $K_{s}$ as the satellite reuse factor. The total number of "pre-design" resources availabe to the system is $\mathrm{M}$, where,

and,

$$
\mathrm{M}=\Sigma_{i=1}^{K_{c}} M_{c_{i}}+\Sigma_{j=1}^{K_{s}} M_{s_{j}}
$$

$M_{c_{i}}=$ number of channels dedicated to cell $C_{i}$.

$M_{s_{j}}=$ number of channels dedicated to spot-beam $s_{j}$.

A mobile user can alternatively access the satellite directly (not through its base station) using a dual mode mobile terminal. New calls are assigned to either layer according to a randomized assignment rule[4]. In this model, we assume a Cellular First [4] assignment rule. All call types have the same priority and all calls considered in this model are mobile-to-mobile calls. Base stations and spot-beams are assumed to be stationary. A mobility model similar to the one employed in the previous section is assumed.

Our main objective here is to show how jointly optimizing the the cell size, relative to the footprint size, and the bandwidth partitioning between the two layers could improve the system performance as compared to the pure cellular alternative. The optimization is to be carried out for given satellite and cellular reuse factors, call assignment policy, and new call arrival and hand-off processes. Accordingly, the optimization problem is formulated as follows,

$$
\min _{n, M_{c_{i}}, M_{s_{j}}} P_{b}
$$


s.t.

$$
\begin{gathered}
P_{f} \leq \beta \\
\mathrm{M}=\Sigma_{i=1}^{K_{c}} M_{c_{i}}+\Sigma_{j=1}^{K_{s}} M_{s_{j}}
\end{gathered}
$$

It is worth mentioning here that we are restricting ourselves to bandwidth partitioning policies having equal bandwidth shares among cells and among spot-beams. This is due to the spatial symmetry of the call arrival rates, service rates, and hand-off rates.

In the following, we consider decomposing the overall optimization problem into simpler sub-problems each of which addresses one aspect of the overall, original problem. The reason we chose to do so is the sheer complexity of the overall problem. The subproblem of finding the optimum channel partitioning, for a given number of cells per spot-beam, has been addressed in [4]. Therefore, we address here the other sub-problem.

Given $M_{c_{i}}$ and $M_{s_{j}}$, the objective is to solve the following minimization problem:

$$
\min _{n} P_{b}
$$

s.t.

$$
P_{f} \leq \beta
$$

This problem is solved for the system having numerical parameters given in Table 2 . In addition, we assume that the satellite reuse factor $K_{s}=2$. Moreover the channel partitioning policy was assumed to be:

$$
M_{c_{i}}=6 \quad, \quad M_{s_{j}}=29
$$

Figure 5 shows the trends of the QoS parameters versus n. Again, it can be noticed that the blocking and dropping probabilities decrease with $\mathrm{n}$. On the other hand, the forced termination probability increases with n. From Figures 4 and 5, we conclude that the hybrid system has better capacity and forced termination performance for the same number of resources.

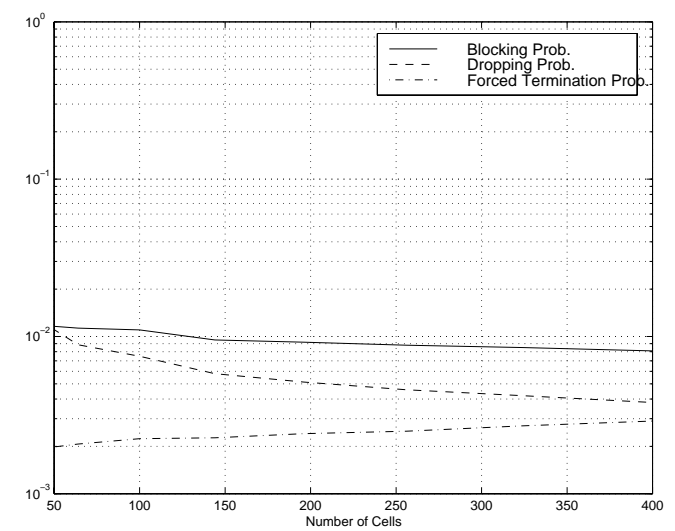

Figure 5. QoS Parameters vs. Number of Cells for a Hybrid System: Simulation Results

To solve the optimization problem, we assume the value of $\beta$ to be 0.0024 . From Figure 5 , it can be noticed that the minimum achievable blocking probability under this constraint is 0.0095 . This is achievable via deploying 36 hexagonal cells underlying each spot-beam.

\section{CONCLUSIONS}

In this paper we studied, analytically and via simulation, the effect of reducing the cell size on the system capacity and hand-off failure rate. First, we focused on pure cellular systems. The exact and approximate results indicate that, for low to moderate mobility, the blocking and dropping probabilities decrease as the number of cells increases. On the other hand, the forced termination probability increases, with the number of cells, for lightly and heavily loaded systems. This trade-off motivated the problem of optimizing the system performance with respect to the cell size. It was noticed that the optimum number of cells depends on the hand-off rate, and the frequency reuse factor, along with other system parameters. Second, we studied a satellite/cellular hybrid system. We noticed that the trends are similar, to a great extent, to those obtained for pure cellular systems. The only difference is the relative improvement of the hybrid system over the pure cellular one. We considered optimizing the hybrid system with respect to the cell size given a channel partitioning policy. We found out that these two degrees of freedom give more flexibility in the system design.

\section{REFERENCES}

(1) Lee, W.C.Y., "Smaller Cells for Greater Performance," IEEE Communications Magazine, Nov. 1991.

(2) Sarnecki, J., Vinodrai, C., Javed, A., O'kelly, P., and Dick, K., "Microcell Design Principles," IEEE Communications Magazine, April 1993.

(3) Wang, L., Stuber, G., and Lea, C., "Architecture Design, Frequency Planning, and Performance Analysis for a Microcell/Macrocell Overlaying System, "IEEE Transactions on Vehicular Technology, vol. 46, no. 4, pp. 836848, Nov. 1997.

(4) ElBatt, T. and Ephremides, A., "Optimization of Connection Oriented, Mobile, Hybrid Network Systems," IEEE Journal on Selected Areas in Communication, vol. 17, No. 2, Feb. 1999.

(5) Rappaport, S., "The Multiple-Call Hand-off Problem in High-Capacity Cellular Communication Systems," IEEE Transcations on Vehicular Technology, vol. 40, no. 3, pp. 546-557, August 1991.

(6) Nanda, S., "Teletraffic Models for Urban and Suburban Microcells: Cell Sizes and Handoff Rates," IEEE Transactions On Vehicular Technology, vol. 42, no. 4, pp. 673-682, Nov. 1993.

(7) Bertsekas, D. and Gallager, R., Data Networks. New Jersy:Prentice-Hall Inc., 1987 (2nd Ed. 1992). 